\title{
Exploring the relationship between Nutrition, gUT microbiota, and BRain AgINg in community-dwelling seniors: the Italian NutBrain population-based cohort study protocol
}

\author{
Federica Prinelli ${ }^{1,2^{*}}$ (D), Nithiya Jesuthasan ${ }^{1}$, Marco Severgnini ${ }^{1}$, Massimo Musicco ${ }^{1 \wedge}$, Fulvio Adorni ${ }^{1}$,
} Maria Lea Correa Leite ${ }^{1}$, Chiara Crespi ${ }^{3}$ and Sara Bernini ${ }^{2}$

\begin{abstract}
Background: Epidemiological evidence suggests that healthy diet is associated with a slowdown of cognitive decline leading to dementia, but the underlying mechanisms are still partially unexplored. Diet is the main determinant of gut microbiota composition, which in turn impacts on brain structures and functions, however to date no studies on this topic are available. The goal of the present paper is to describe the design and methodology of the NutBrain Study aimed at investigating the association of dietary habits with cognitive function and their role in modulating the gut microbiota composition, and brain measures as well.

Methods/design: This is a population-based cohort study of community-dwelling adults aged 65 years or more living in Northern Milan, Italy. At the point of presentation people are screened for cognitive functions. Sociodemographic characteristics along with lifestyles and dietary habits, medical history, drugs, functional status, and anthropometric measurements are also recorded. Individuals suspected to have cognitive impairment at the screening phase undergo a clinical evaluation including a neurological examination and a Magnetic Resonance Imaging (MRI) scanning (both structural and functional). Stool and blood samples for the gut microbiota analysis and for the evaluation of putative biological markers are also collected. For each subject with a confirmed diagnosis of Mild Cognitive Impairment (MCl), two cognitively intact controls of the same sex and age are visited. We intend to enrol at least 683 individuals for the screening phase and 240 persons for the clinical assessment.

\footnotetext{
* Correspondence: federica.prinelli@itb.cnr.it

Massimo Musicco is deceased.

'Institute of Biomedical Technologies-National Research Council, Via Fratelli Cervi, 9320090 Segrate, Ml, Italy

${ }^{2}$ IRCCS Mondino Foundation, Neuropsychology/Alzheimer's Disease Assessment Unit, Via Mondino 2, 27100 Pavia, Italy

Full list of author information is available at the end of the article
}

C C The Author(s). 2020 Open Access This article is licensed under a Creative Commons Attribution 4.0 International License, which permits use, sharing, adaptation, distribution and reproduction in any medium or format, as long as you give appropriate credit to the original author(s) and the source, provide a link to the Creative Commons licence, and indicate if changes were made. The images or other third party material in this article are included in the article's Creative Commons licence, unless indicated otherwise in a credit line to the material. If material is not included in the article's Creative Commons licence and your intended use is not permitted by statutory regulation or exceeds the permitted use, you will need to obtain permission directly from the copyright holder. To view a copy of this licence, visit http://creativecommons.org/licenses/by/4.0/ The Creative Commons Public Domain Dedication waiver (http://creativecommons.org/publicdomain/zero/1.0/) applies to the data made available in this article, unless otherwise stated in a credit line to the data. 
(Continued from previous page)

Discussion: The NutBrain is an innovative study that incorporates modern and advanced technologies (i.e. microbiome and neuroimaging) into traditional epidemiologic design. The study represents a unique opportunity to address key questions about the role of modifiable risk factors on cognitive impairment, with a particular focus on dietary habits and their association with gut microbiota and markers of the brain-aging process. These findings will help to encourage and plan lifestyle interventions, for both prevention and treatment, aiming at promoting healthy cognitive ageing.

Trial registration: Trial registration number NCT04461951, date of registration July 7, 2020 (retrospectively registered, ClinicalTrials.gov).

Keywords: Cognitive impairments, Dietary habits, Observational study, Gut microbiota, Brain measures, Gut-brain axis

\section{Background}

Human aging can be viewed as a complex and multifactorial phenomenon resulting from an interaction between genetic background, environmental factors, epigenetic and stochastic events [1]. Aging is the main risk factor for the development of several non-communicable diseases such as cancer, diabetes, cardiovascular and neurodegenerative diseases. Since population grows older, we expect an increase in the occurrence of these age-related diseases [2]. Among them, dementia and cognitive impairment are becoming leading causes of disability in the older population [3]. In 2015, about 47 million people were affected by dementia worldwide. In European countries, the prevalence is about $6.4 \%$ in people aged 65 years or older [4] with an incidence that doubles every 5.9 years, increasing with population age, varying from 3.1 cases per 1000 personyears in the age group 60-64 to 175 cases per 1000 years person in the age group above 95 years [5]. The transitional phase between normal physiological and pathological aging is a clinical condition called Mild Cognitive Impairment (MCI), characterized by a deficit in one or more cognitive domains (memory, visual-spatial and executive function, attention, and language) without compromising the normal daily activities [6]. The data recently published by the COSMIC International Consortium report a prevalence of MCI in subjects aged 60 or more ranging from 7 to $21 \%[7,8]$, with an incidence that varies from 51.0 to 76.8 cases per 1000 person-years in our country [9]. Since about $15 \%$ of MCI subjects yearly converts to dementia [10], it is important to better characterize this group of people in order to intervene in a timely manner.

To date, only limited, primarily symptomatic treatment options are available and do not have proven effects on delaying disease progression. Thus, there is a growing interest in identifying non-pharmacological strategies able to prevent or delay the onset of disease [11]. Age, sex, family history of disease and genetic susceptibility (i.e. carriers of the Apolipoprotein E $\varepsilon 4$ genotype - APOE $\varepsilon 4$ ) are known but not modifiable risk factors. Therefore, an intervention on modifiable risk conditions such as hypertension, diabetes, dyslipidaemia, obesity, neuropsychiatric symptoms, and poor lifestyles (unhealthy diet, low cognitive stimulation, physical inactivity, smoking habit, and limited social network) seems to be the most promising approach [12].

Accumulating epidemiological evidence suggests that intake of specific nutrients including antioxidants (vitamin $\mathrm{C}, \mathrm{E}, \mathrm{A}$, and carotenoids), long-chain polyunsaturated fatty acids (PUFAs - n3) and B vitamins (B9, B6, B12) is associated with a slowdown of cognitive decline and a reduced risk of dementia [13]. Despite the encouraging results from observational studies, clinical trials on vitamins and supplements report conflicting results [14], mostly because of the complexity of dietary exposure. In order to take into account for the biological interactions between different components of the food "matrix", several dietary patterns, such as the Mediterranean Diet (MD)-type pattern or other "healthy" indices [15-17], have been developed and investigated in relation to cognitive disorders. The majority of the epidemiological studies reported a reduction in the risk of cognitive impairment for increasing adherence to MD and "Prudent" pattern, characterized by foods of plant origin, fish, poultry, and whole grains. On the contrary, the "Western" pattern, based on red and processed meat, fats, refined cereals, snacks, sugars, alcohol and a reduced fibre content, is associated with an increased risk of cognitive disorders [18-21]. Currently, a few multi-domain intervention studies including dietary components (e.g. the PREDIMED [22, 23], preDIVA [24], MAPT [25], FING ER [26] and other randomized controlled trials (RCTs) [27-29]) are performed in cognitively healthy older persons or in people at risk of developing dementia [30, 31].

The biological mechanisms through which the various dietary components could exert a protective effect on the brain mainly involve processes linked to reduce oxidative stress [32], mitochondrial function, immune dysfunction, inflammatory state, and altered nutrientsensing mechanisms [33]. However, the exact nature of these interactions and the underlying mechanisms are poorly explored and understood.

In recent decades, research has highlighted the potential role of the human gut microbiota in the regulation 
of the immune system, in the absorption of nutrients, as well as in the physiology of the nervous system and brain functions [34]. Moreover, alterations to the composition of the bacterial population of the human gut have been associated with various pathological conditions in the host including some neurodegenerative disorders such as Parkinson's disease [35] and cognitive impairment [36,37]. Diet represents one of the main determinants of gut microbiota due to its ability to modulate the microbe population composition, which in turn, impacts on the host therefore;, a dietary intervention can be considered a valid approach in the prevention and/or treatment of these diseases [38]. Within the bidirectional interactions of the gut-brainaxis, the gut microbiome communicates to the central nervous system primarily through neuroendocrine and neuro-immune signalling mechanisms and, also, via the generation of bacterial metabolites, which exert their physiologic effects both locally and systemically [39]. Despite considerable progresses, the knowledge in this area is scarce and the mechanisms underlying this relationship are still overlooked.

An innovative approach in understanding the impact of human gut microbiota on brain health, involves the use of neuroimaging, which would foster the identification of potential mediators of this relationship [40, 41]. Furthermore, recent studies have shown that brain imaging, given the precision in measuring changes in the structures and functions of the brain associated with the aging process, can be a reliable tool for exploring the relationship between diet, gut microbiota, and brain measures [42, 43]. The ground-breaking hypothesis underlying the present study is that the strict connection between diet, gut microbiota, and brain can partly explain how our dietary habits may accelerate or slow down brain aging. Bearing these considerations in mind, the NutBrain Study (Exploring the relationship between Nutrition, gUT microbiota, and BRain AgINg) aims to understand the biological mechanisms through which diet influences cognitive disorders with a special focus on the impact of nutrition on gut microbiota and brain characteristics, by applying a novel multi-level approach that integrates traditional epidemiological methods with neuroimaging and gut microbiota profiling. Aims of the NutBrain study are: i) to estimate the occurrence of MCI and other cognitive disorders in community-dwelling older people aged 65 + years; ii) to investigate the association between lifestyle habits and cognitive ageing outcomes; iii) to explore the role of diet, in modulating the gut microbiota composition, which in turn impacts on brain structures and functions as well.

The goal of the present paper is to describe the design and methodological approach of the NutBrain study protocol.

\section{Methods}

Study design, setting, and participants

The NutBrain Study is an ongoing population-based cohort study promoted by the Institute of Biomedical Technologies of the National Research Council (ITBCNR, Segrate, Italy) and the Hospital IRCCS Fondazione Mondino (Pavia, Italy). The NutBrain Study is structured in three phases (Fig. 1).

\section{$T=1$ Participant's recruitment}

The study is performed in two sites in the outskirt of North-Milan: Bollate and Baranzate. A random sample is drawn from the official register of residents in the two municipalities. Inclusion criteria are: attending a medical appointment in the research facility, living at home in one of the two municipalities, and being $65+$ years. Eligible population is contacted by means of a letter of invitation, in which people are invited to contact the ITB-CNR by phone to schedule the first visit during which participant is asked to bring last instrumental and clinical exams prescribed by their general practitioner. A comprehensive communication campaign is implemented to publicize and promote the project as well as to foster recruitment. To ensure a high enrolment rate, the Mayors of the two municipalities have been actively involved since the earliest planning stages of the recruitment and have granted patronage to the project. Additionally, the study is promoted and advertised at the community level through different channels: by printing brochures and setting posters in senior recreation centres, shops, pharmacies, medical doctors office, and patients associations; by articles and advertisements in local newspapers, institution websites, as well as in social media (e.g. Facebook, https://www.facebook.com/TheNutBrainStudy/) [44]. The NutBrain Study has a website (www.nutbrain.it) where potential participants are directed for further information and can register interest to participate. In addition, to raise awareness, several public events describing the objectives of the proposed study are organized for recruiting eligible people. $\mathrm{T}=1$ started in October 2018 .

\section{$T=2$ screening evaluation}

Data collection started in April 2019. Participants are visited at the research facility in their residence town (arranging home visits for disabled individuals) by a trained team. Informed consent form is completed at the research facility prior to data collection. In those individuals without capacity to give full informed consent, proxy consent is collected from relatives or caregivers. This 2-h interview includes a face-to-face administration of a neuropsychological battery of tests and questionnaires to inquire about socio-demographic, occupational, and social-economic data, education, medical conditions and drug use, lifestyle habits, functional status, and 


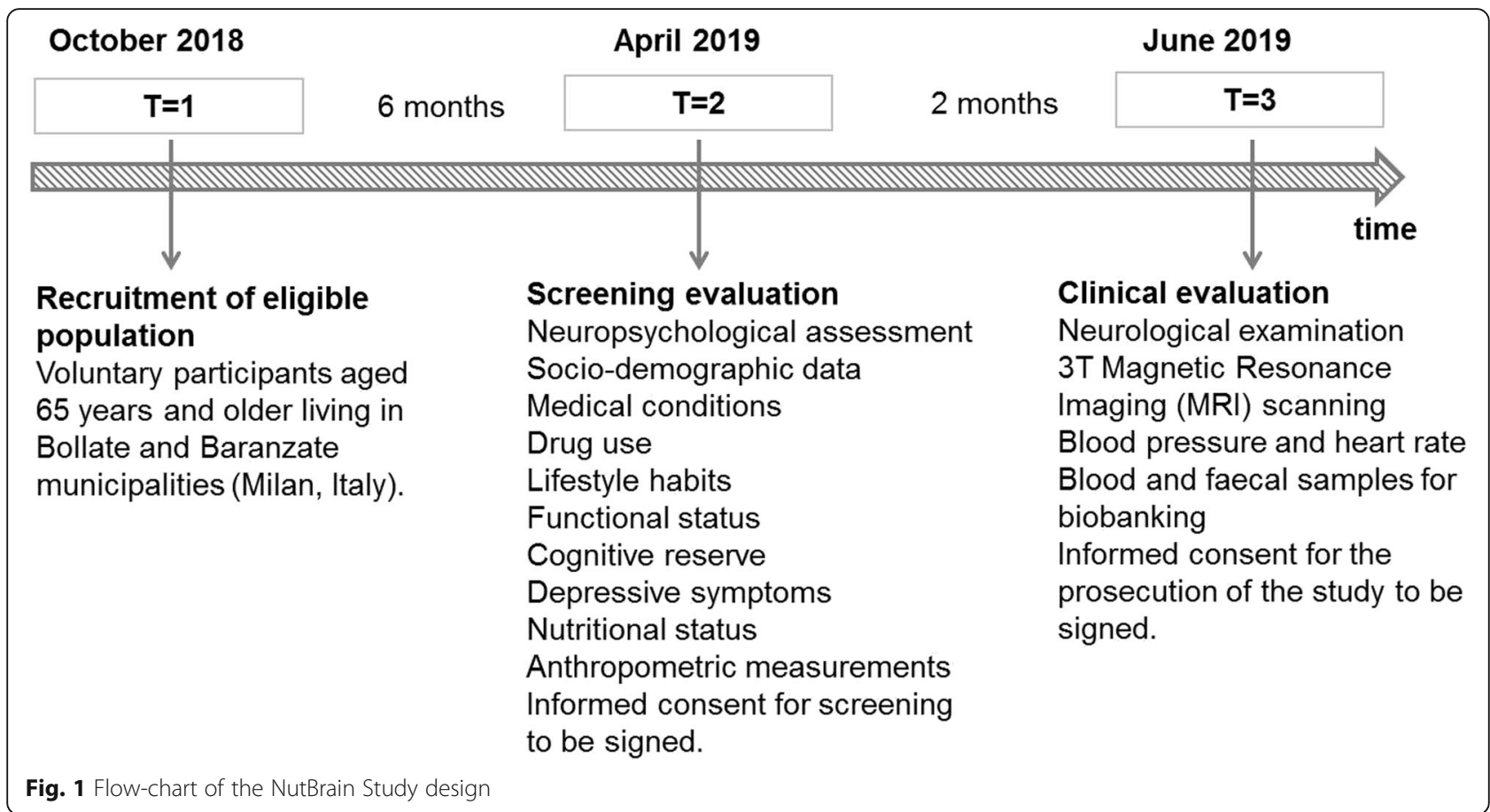

dietary behaviours. At the end of the visit, a stool sampling kit consisting of a sterile faeces container with instructions explaining the procedure for the stool sample collection, and a 3-day food diary is provided to each participant. The first visit serves to screen each participant for cognitive functions and for identifying inclusion/exclusion criteria in a more comprehensive manner.

\section{$T=3$ clinical evaluation}

Individuals suspected to have cognitive impairment at the screening neuropsychological tests undergo a clinical examination. Participants are transported from their own home to the Hospital using a private transport service, whose cost is covered entirely by the study. The hospital visit includes a standard neurological examination performed by a neurologist and a $3 \mathrm{~T}$ Magnetic Resonance Imaging (MRI) scanning. At this stage, blood and stool samples are collected from each participant and stored in the biorepository at the Hospital until processing. For each subject with a clinical diagnosis of MCI two cognitively intact controls of the same sex and of the closest date of birth are enrolled and visited at the Hospital and undergoing the same protocol as MCI individuals. $\mathrm{T}=$ started in June 2019 .

\section{Ethics approval and consent to participate}

The study protocol and paperwork have been reviewed and approved by the Medical Ethical Committee of Pavia, Italy (Ref. number: 20180036036, 2018/04/20 and amendment Ref. Number: 20190045757, 2019/05/21). All participants provide a formal written informed consent in order to participate in the study according to the Declaration of Helsinki. In those individuals found to be without capacity to give full written informed consent, a caregiver or guardian is identified and their advice sought regarding participation.

\section{Data collection}

A comprehensive protocol has been designed for this study. Table 1 gives an overview of the variables that are collected in the NutBrain project and the corresponding tools used for assessment.

\section{Socio-demographics, medical, and lifestyle data}

At $\mathrm{T}=2$, participants are asked about their sociodemographic characteristics including education, marital status, work history and retirement, as well as living situation (i.e. co-habitants). Information regarding the house property and the number of rooms are used as an indicator of the household income. Participants also provide information about all current and past medical conditions, surgery and illnesses, use of medications, supplements, and hospitalizations. A detailed menstrual gynecological history is obtained from female participants (age at menarche and menopause, birth-control pill and hormones replacement therapy use, and number of births and breastfeeding). Information regarding the medical history of the participant's first degree relatives is also collected. A series of questions are used to assess type and frequency of smoking habit. Assessment of physical activity is performed using the validated International Physical Activity Questionnaire (IPAQ) [45], which includes 7 items, divided into 
Table 1 Summary of assessments and testing instruments used in the NutBrain Study

\begin{tabular}{|c|c|}
\hline Variables & Instruments/assessment tools \\
\hline \multicolumn{2}{|l|}{ Questionnaires } \\
\hline Health and socio-demographics & $\begin{array}{l}\text { Written questionnaire developed by the researchers regarding: socio-demographic information, } \\
\text { household income, family history of diseases, medical conditions, surgery and illnesses, use of } \\
\text { drugs and supplements, hospitalization, menstrual gynaecological history, and smoking habit. }\end{array}$ \\
\hline Physical Activity level & International Physical Activity Questionnaire (IPAQ) [45] \\
\hline Cognitive reserve & Cognitive Reserve Index questionnaire (CRlq) [46] \\
\hline \multirow[t]{2}{*}{ Functional status } & Instrumental Activities of Daily Living scale (IADL) [47] \\
\hline & Katz Index of Independence in Activities of Daily Living (ADL) [48] \\
\hline Depression & Center for Epidemiologic Studies Depression (CES-D) scale \\
\hline \multicolumn{2}{|l|}{ Anthropometry } \\
\hline Height & Portable wall-mounting stadiometer SECA 213 \\
\hline Weight & Homologated electronic scale Tanita SC240MA \\
\hline Waist and mid-upper arm circumferences & Flexible graduated measuring tape SECA 201 \\
\hline Fat mass (FM\%) and Total Body Water (TBW\%) & Bioelectrical impedance analysis (BIA) (Tanita SC240MA) \\
\hline \multicolumn{2}{|l|}{ Biological samples } \\
\hline Blood & Cells, plasma and genomic DNA (for APOE genotyping) \\
\hline Stool & $\begin{array}{l}\text { Alpha- and beta-diversity measures, bacterial relative abundances (indexes estimated on the } \\
16 \mathrm{~S} \text { rRNA-based sequencing data) }\end{array}$ \\
\hline \multicolumn{2}{|l|}{ Clinical } \\
\hline Blood pressure & Sphygmomanometer \\
\hline Heart rate & Heart rate monitor \\
\hline \multicolumn{2}{|l|}{ Neuroimaging } \\
\hline MRI scans & Siemens MAGNETOM 3 T scanner \\
\hline \multicolumn{2}{|l|}{ Nutritional status } \\
\hline \multirow[t]{2}{*}{ Dietary habits } & Semi-quantitative food frequency questionnaire (SFFQ) [49] \\
\hline & Estimated 3-day food diary \\
\hline Malnutrition & Mini-nutritional assessment (MNA) [50] \\
\hline \multicolumn{2}{|l|}{ Neuropsychological assessment } \\
\hline Global cognitive function: & MMSE [51] \\
\hline \multicolumn{2}{|l|}{ Cognitive domains } \\
\hline \multirow[t]{3}{*}{ Memory } & Free and Cues Selective Reminding Test (FCSRT) [52] \\
\hline & Logical Memory test - Babcock Test [53] \\
\hline & Rey-Osterrieth Complex Figure Test (ROCF) - delay recall [54] \\
\hline \multirow[t]{3}{*}{ Execute function } & Frontal Assessment Battery (FAB) [55] \\
\hline & Phonemic and semantic verbal fluency [56] \\
\hline & Trial Making Test (TMT A and B) [57] \\
\hline Language & Picture Naming Test [58] \\
\hline Visuo-spatial abilitities & Rey-Osterrieth Complex Figure Test (ROCF) - copy [54] \\
\hline
\end{tabular}

frequency, intensity, and duration of physical activity at low (walking), moderate, and vigorous levels, in addition to total physical activity during the last week. It also includes an item about sitting time, expressed as minutes per day, measuring sedentary lifestyle on weekdays and weekend days. Functional evaluations of activities of daily living are assessed using the Katz Index of Independence in Activities of Daily Living (ADL) [48]. The index ranks adequacy of performance in the six functions of bathing, dressing, toileting, transferring, continence, and feeding. Participants are scored yes/no for independence in each of the six functions. The participants' ability to use the telephone and transportation, medication management and handling of finances independently is assessed using the Instrumental 
Activities of Daily Living scale (IADL) [47]. Participation in intellectual, social, physical and leisure activities is measured using the Cognitive Reserve Index questionnaire (CRIq), which includes 20 items grouped into 3 sections: education, working activity, and leisure time, each returning a sub-score [46]. Center for Epidemiologic Studies Depression (CES-D) scale is used to measure symptoms associated with depression experienced in the last week [59].

\section{Anthropometric and clinical measurements}

A trained dietician measures participants' body weight, height, fat mass, total body water, and waist and mid-upper arm circumferences during the screening evaluation. Body weight (in $\mathrm{Kg}$ to the closest $0.5 \mathrm{~kg}$ ) and body composition (Fat mass [FM\%] and Total Body Water [TBW\%]) are assessed by bioelectrical impedance analysis (BIA) measured using a homologated leveled-platform electronic scale (Tanita SC-240MA), with participants wearing light clothing and no shoes. Height (in $\mathrm{cm}$ to the closest $0.5 \mathrm{~cm}$ ) is measured with a portable wall-mounting system with the participants shoeless in the standing position (SECA 213). Body Mass Index (BMI) is calculated by the standard formula (weight/height ${ }^{2}$ ). Waist circumference and mid-upper arm circumference are measured in the middle between the 12th rib and the iliac crest, and the midpoint between the tip of the shoulder and the tip of the elbow, respectively (in $\mathrm{cm}$ closest to the $0.5 \mathrm{~cm}$ ). Body circumferences are measured using a flexible graduated measuring tape (SECA 201) with the participant in the standing position. During the clinical evaluation, a nurse measures systolic and diastolic blood pressure and heart rate with the participants in the sitting, resting position using a sphygmomanometer and a heart rate monitor, respectively.

\section{Dietary assessment}

A trained dietician performs a detailed nutritional assessment. A semi-quantitative food frequency questionnaire (SFFQ) (adapted from the validated Willet's questionnaire) [49] is used to collect dietary habits over the prior year. Participants are asked to indicate how often they consume a standard portion of a given item according to a nine-category frequency scale: never/seldom (lessthan once per month), 1-3 times per month, 3 times per week, 2-4 times a week, 5-6 times a week, once a day, 2-3 times a day, 4-5 times per day, and 6+ times a day. Color pictures of serving sizes of each food item are showed to help in the understanding of standard portion sizes. Participants enrolled in the third phase of the study $(\mathrm{T}=3)$ are asked to keep a food diary to record a detailed description of types and amounts of food and beverages consumed over a period of 3 days before the fecal sample collection including two weekdays and one weekend day. To convert frequency data into daily energy and macro- and micronutrient intake, frequencies are multiplied by standard serving sizes using the Italian Food Composition Databases for Epidemiological studies in Italy (http://www.bda-ieo.it/) and the MetaDieta software (Meteda s.r.l., Ascoli Piceno, 227 Italy). The MiniNutritional Assessment (MNA) is administered as a screening tool to identify malnourished individuals or those at risk of malnutrition [60].

\section{Neuropsychological assessment}

The neuropsychological tests have been selected according to the guidelines for the diagnosis of MCI [50]. Current conceptualizations of MCI recognize multiple subtypes centered on the presence or absence of memory impairment, namely amnestic (aMCI) and non-amnestic (naMCI) and on the number of compromised cognitive domains (MCI single domain vs MCI multiple domain) (Fig. 2) [61]. To this scope, an experienced neuropsychologist administers the following battery of well-established neuropsychological tests exploring global cognitive function (Mini Mental State Examination-MMSE) [51] and different cognitive domains: memory (Free and Cues Selective Reminding Test (FCSRT) [52], Logical memory test - Babcock Test [53], the Rey-Osterrieth Complex Figure Test (ROCF) delay recall [54], executive function (Frontal Assessment Battery (FAB) [55], phonemic and semantic verbal fluency [56], Trial Making Test (TMT) [57], language (Picture Naming Test [58]), visuo-spatial abilities (Rey-Osterrieth Complex Figure Test (ROCF) - copy [54]). All the test scores are corrected for age, sex, and education and compared with the values available for the Italian population (see Supplementary material).

\section{Inclusion/exclusion criteria}

Screened participants are selected among all subjects willing and able to undergo all test procedures including neuroimaging, blood and stool sample collection. General inclusion/exclusion criteria are the following:

i) neuropsychological criteria: we enrol subject with different subtypes of $\mathrm{MCI}$ (aMCI, naMCI, single-domain MCI, multiple-domain $\mathrm{MCI}$ ) according to Albert's criteria [50] and subject with normal cognitive profile. Subject with dementia, pre-existing cognitive impairment (e.g. aphasia, neglect), concomitant severe psychiatric disease, others neurological conditions (e.g. severe depression and behavioral disorders) or severe sensory disturbances (e.g. auditory and/or visual loss) that do not allow completing neuropsychological assessment are excluded from the third phase of the protocol.

ii) microbiota' analysis protocol: we exclude individuals with artificial nutrition in progress; those with a history of active uncontrolled gastrointestinal disorders or diseases, including inflammatory bowel disease, ulcerative colitis, and Crohn's disease; subjects who underwent 


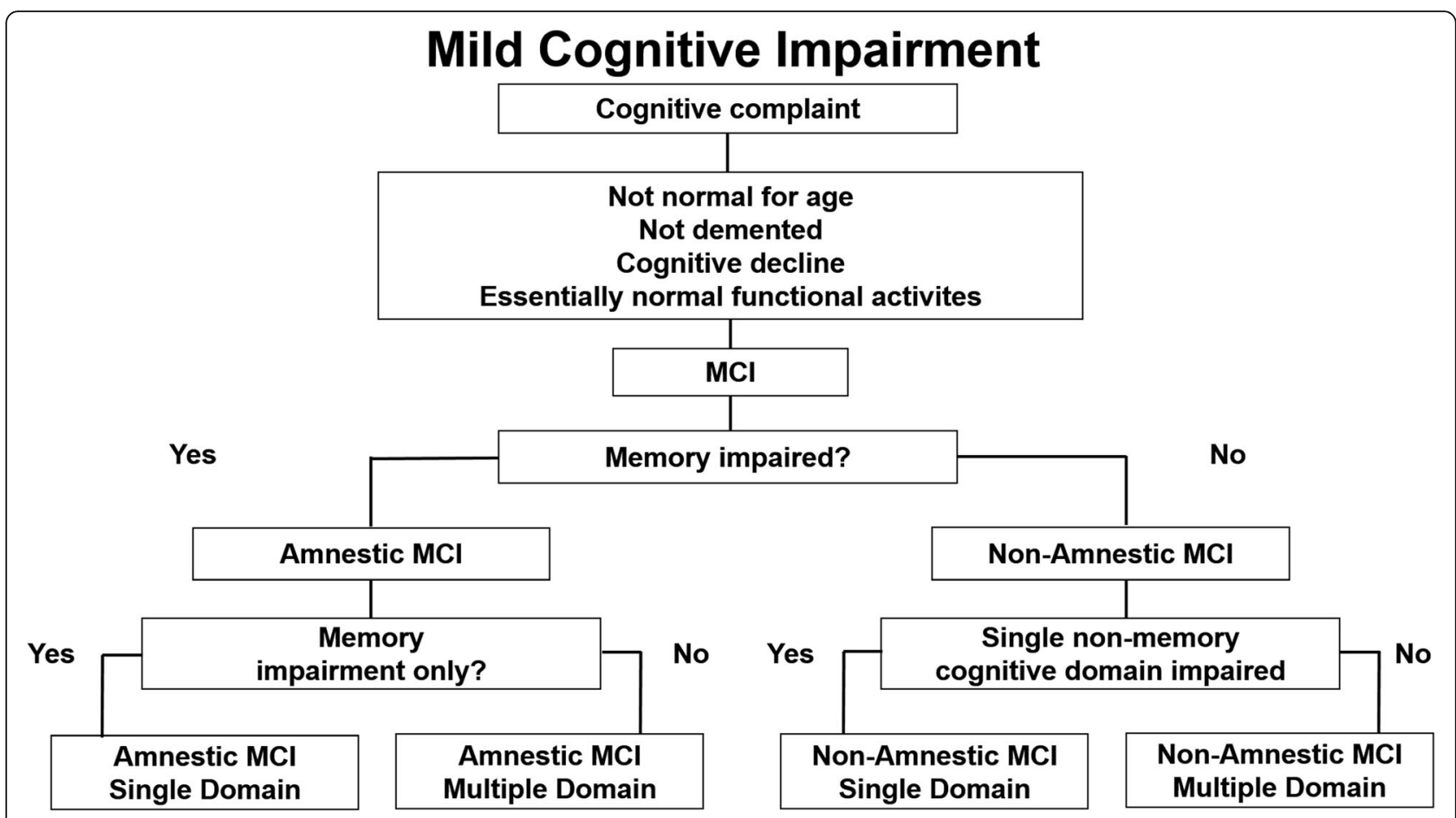

Fig. 2 Algorithm used to classify the subtypes of Mild Cognitive Impairment (MCI) (Source: Petersen R [51].

previous major surgery on the gastro-enteric tract, with the exception of cholecystectomy and appendectomy, in the past five years, use of antibiotics or large doses of commercial probiotics in the 4 weeks prior the visit, and subjects under radio-chemo-therapy.

iii) MRI scanning protocol: we exclude those with metal fragments in the body, surgically implanted devices containing metal, severe claustrophobia, or inability to lie down in the MRI scanner for the duration of the study.

\section{Endpoint determination}

Once the presence and type of MCI have been defined by neuropsychological assessment, a certified neurologist performs the neurological assessment in order to recognize the possible cause of the clinical syndrome through a careful anamnestic investigation. To meet the core clinical criteria for $\mathrm{MCI}$, it is necessary to rule out other systemic or brain diseases that could account for the decline in cognition (e.g., vascular, traumatic, medication side effects). The goal of such an evaluation is to increase the likelihood that the underlying disease is a neurodegenerative disorder. A final diagnosis is assigned by the neurologist after reaching an agreement between the neuropsychological and clinical examinations.

\section{MRI measures}

Neuroimaging data are acquired, pre-processed and analyzed at the Hospital. MRI data are acquired using a 3 Tesla MAGNETOM scanner (Siemens, Erlangen,
Germany). MRI measures include structural (high resolution T1 anatomical scan - grey matter volume/density, cortical thickness; Diffusion Tensor Imaging scan: white matter microstructural integrity) and functional characteristics (resting state fMRI sequence: functional connectivity at rest). Preprocessing and voxelwise analysis of grey and white matter characteristics, as well as functional connectivity analysis, are carried out using appropriate toolboxes. We perform: i) standard univariate (hypothesis-driven) analyses of grey and white matter features (e.g., voxelbased morphometry, cortical thickness, tract-based spatial statistics) to obtain macrostructural and microstructural metrics discriminating patients from controls; ii) multivariate data-driven analyses of neurofunctional features (i.e., functional connectivity) and multi-modal data (i.e., integration of structural and functional features) to investigate group differences at a network level; and iii) network analysis to identify distinctive characteristics of the structural and functional connectomes in patients and controls. MRI scans are labeled according to each site's imaging capabilities using code subject identifiers. All scans undergo a deidentification process to ensure that no subject's identification is present in the images files.

\section{Biological samples for biobanking}

Blood sample. A qualified nurse draws $30 \mathrm{ml}$ of blood sample (non-fasting state). Blood (cells and plasma) and genomic DNA (for APOE genotyping) are collected 
using EDTA tubes and are stored at $-80{ }^{\circ} \mathrm{C}$ in the Hospital's biorepository according to international consensus standard operation procedures. Samples will be stored for 25 years for potential future analysis. Polymerase Chain Reaction (PCR) is performed as described by Wenham et al. [62] and the final products are digested with restriction enzyme for assessing the APOE polymorphic sites.

Stool sample. Participants are asked to provide a stool sample (about $250 \mathrm{mg}$ ) that is immediately frozen at $20^{\circ} \mathrm{C}$ and, later, stored at $-80^{\circ} \mathrm{C}$ in the biorepository until processing. Total bacterial DNA is extracted using the QIAamp DNA Stool Mini Kit (QIAGEN, Valencia, CA, USA) with a modified protocol [63] based on beadbeating with glass and zirconia beads in a FastPrep-24 Instrument (MP Biomedicals, Santa Ana, CA, USA). DNA concentration and quality for each sample is determined using a NanoDrop ND-1000 spectrophotometer (NanoDrop Technologies, Wilmington, DE, USA). For each faecal sample, the V3-V4 regions of the microbial $16 \mathrm{~S}$ rRNA gene are PCR-amplified using primers carrying specific overhang adapters appended to the primer pair sequences for compatibility with Illumina index and sequencing adapters. Taxonomy analysis are performed within the QIIME suite [64] grouping reads into Operational Taxonomic Units (OTUs) at 97\% similarity and classifying them against the Greengenes database (ftp:// greengenes.microbio.me/greengenes_release/gg_13_8_otus) through RDP classifier [65] Alpha-diversity (i.e.: species diversity within samples) is evaluated calculating Chaol, Shannon's index, total number of OTUs and Faith's phylogenetic diversity metrics, whereas beta-diversity (i.e.: diversity in bacterial composition among the samples) is estimated through weighted and unweighted Unifrac distances [66] Bacterial composition of the samples in terms of relative abundance is compared at all phylogenetic levels down to genus and differences are evaluated by nonparametric Mann-Whitney U-test for equal medians. Statistical evaluation among alpha-diversity indices is performed by a non-parametric Monte Carlo-based test, using random permutations. "adonis" function of the R package "vegan" (https://cran.r-project.org/web/packages/vegan/index.html) with random permutations is employed to determine statistical separation of the microbiota profiles.

\section{Data management and confidentiality}

Data are handled, monitored, computerised, and stored in accordance with the European General Data Protection Regulation (EU) 2016/679 (GDPR) (https://gdprinfo.eu/). All study records, including the consent forms, are kept in a locked filing cabinet at the ITB-CNR where the file server for data storage is located. The file server is firewalled within the ITB-CNR intranet. For privacy and security, a password granted only to the server administrator is required to access to the database. The data forms are double checked for missing data and inconsistencies. Quality of the database entered data is monitored by checking entry for a random sample of participants. Data transfer is protected by means of crypting/decrypting policy and password protection. In the final dataset a unique key identifies each subject to guarantee anonymity. Personal data are regarded as strictly confidential and removed before the exportation procedure. Security of data is guarantee via automatic backups.

\section{Sample size}

Given the observational nature and the "exploratory" objectives of the study design, and the large number of factors to be analyzed, formal sample size calculations for a specific aim are not feasible. However, considering an expected prevalence of individuals with MCI of approximately $20 \%$ in the general population aged over 65 from this geographical area [67] (population of resident seniors $~ 9000$ ), a desired precision of $3 \%$ and a confidence levels of 95\%, a sample size of 683 is required for the screening phase $(\mathrm{T}=2)$. Conservatively assuming a maximum loss of $40 \%$ of participants to $\mathrm{T}=3$ due to ineligibility or refusal, we expect about $80 \mathrm{MCI}$ individuals undergo phase 3 . For each diagnosed MCI case, two cognitively intact controls $(1: 2$ ratio, $N=160)$ of the same sex and closest in birth date to the case $( \pm 1$ year) are selected from the population under investigation reaching a total of 240 individuals undergoing $\mathrm{T}=3$.

\section{Planned statistical analysis}

For the descriptive analysis of the variables under study, frequencies and proportions are used for qualitative variables, and mean \pm standard deviations, medians \pm interquartile intervals are used for quantitative variables. Subsequently, the differences between subjects with MCI diagnosis and cognitively intact controls are analyzed with respect to various variables of interest (e.g. dietary exposures, brain measures, gut microbiota composition, etc.). Comparisons between continuous variables are carried out using ANOVA tests for independent samples (for normally distributed variables) or non-parametric Mann-Whitney and Kruskall-Wallis tests (for nonnormally distributed variables). Fisher exact test and $x^{2}$ test is used to compare categorical variables. Data are analyzed using several methodological approaches such as data reduction techniques (e.g. clusters or principal component analysis) and Compositional data analysis (CoDa) [68, 69]. Subsequently, the analysis can be extended by applying different multivariate regression models (linear and conditioned logistic) to study the relationship between the exposures and the different outcomes of interest, both in univariate and multivariate 
analysis by adjusting for possible confounders (e.g. socio-demographic variables, other lifestyles, etc.). Test for interaction and stratified analyzes are also performed (e.g. sex, age, or APOE genotype) to assess effect modification. The robustness of the study results are verified by conducting different sensitivity analyzes. The level of significance is set at $\alpha=0.05$ and all tests are two-tailed. Statistical analyzes are performed using the STATA software packages (version 15, StataCorp LP, College station, Texas, USA) and SPSS (IBM Corp. Released, IBM SPSS Statistics version 25.0 Armonk, NY: IBM Corp.).

\section{Dissemination and provision of results to participants}

Dissemination of the scientific data obtained from this project is performed through a detailed dissemination plan that takes into account for the target audience. The results of this study are communicated through peerreviewed journals, national and international congress presentations, academic lessons, workshops, technical journals (for researchers and general practitioners), institutional and private web sites; and disseminated through associations and local printed media. On completion of the study, participants will receive a report summarizing the main results of the NutBrain Study.

\section{Discussion}

In this study we apply an innovative "system epidemiology" [70] approach that integrates traditional epidemiological methods with modern and advanced technologies to provide new insights into the biological mechanisms underlying the relationship between dietary habits and brain aging.

The results of the NutBrain Study may have public health implications at different levels: i) unravelling the mechanisms underlying relationship between diet, gut microbiota, and age-related disorders, ii) improving the scientific knowledge with the aim to identify the best suited population for early and tailored intervention strategies, iii) providing strong evidence-based recommendations and guidelines for promoting a healthy lifestyle in target population, iv) paving the way towards new prospects for precision nutrition and medicine.

From a methodological point of view, due to the observational nature of the study design is difficult to derive causal relationship. Furthermore, our study is prone to certain biases such as reverse causation and selection bias (volunteer bias). Accordingly, the strength of the associations might be underestimated and generalization of our findings to other populations should be done with caution. However, the availability of a well-characterized cohort of elderly from the general population that includes dietary assessment, neuropsychological tests and biological samples collection, together with the integration of advanced techniques (microbiomics and neuroimaging), is a novelty and the main strength of this study. Additionally, the baseline assessment allows setting up future prospective investigation on the potential epidemiological, clinical, and biological determinants of disease progression. Because the pathological process of dementia precedes by decades the severe clinical manifestations of disease, an early intervention in asymptomatic or early mild symptomatic individuals is a promising and cost-effective solution in the agenda priorities of policymakers and governments.

In conclusion, to lessen the burden of age-related diseases and ameliorate quality of life among older people, a better understanding of the intricate mechanistic pathways subjacent brain aging is an urgent need. This "pioneer" study represents a unique opportunity to improve this knowledge, to translate into practice and to devise interventions for both prevention and treatment of age-related cognitive disorders.

\section{Supplementary information}

Supplementary information accompanies this paper at https://doi.org/10. 1186/s12877-020-01652-2.

Additional file 1. Neuropsychological assessment.

\section{Abbreviations}

$\mathrm{MCl}$ : Mild Cognitive Impairment; MRI: Magnetic Resonance Imaging; APOE $\varepsilon 4$ : Apolipoprotein E $\varepsilon 4$ genotype; PUFAs: Polyunsaturated fatty acids; MD: Mediterranean Diet; RCTs: Randomized Controlled Trials;

IPAQ: International Physical Activity Questionnaire; ADL : Index of Independence in Activities of Daily Living; IADL : Instrumental Activities of Daily Living scale; CRlq : Cognitive Reserve Index Questionnaire; CESD: Center for Epidemiologic Studies Depression; FM: Fat mass; TBW: Total Body Water; BIA: Bioelectrical Impedance Analysis; BMI: Body Mass Index; SFFQ: Semi-quantitative Food Frequency Questionnaire; MNA: Mini Nutritional Assessment; MMSE: Mini Mental State Examination; FCSRT: Free and Cues Selective Reminding Test; ROCF: Rey-Osterrieth Complex Figure Test; FAB: Frontal Assessment Battery; TMT: Trial Making Test;

OTUs: Operational Taxonomic Units; CoDa: Compositional Data Analysis

\section{Acknowledgments}

The authors would like to express their sincere appreciation and gratitude to all participants and their families for their participation in the NutBrain Study. A special thank is extended to the Mayors of Bollate and Baranzate, the community of the two municipalities (employees, Centro Anziani di Bollate, Associazione Volontari Divers/Età di Baranzate), and the Cooperativa 'II Grappolo' for their cooperation and practical support. The Bollate municipality also provides the facility "SPAZIO UNICO" where the survey is performed. The authors also would like to gratefully thank the researchers, physicians, technicians, nurses and the administrative staff for their efforts, contributions, and support to the project.

\section{Authors' contributions}

FP, as principal investigator, has full access to all data in the study and takes responsibility for the integrity of the data. She conceived and designed the study, acquired funding and ethics approval. She wrote the original draft of the manuscript. NJ, MS, MM, FA, MLCL, CC, and SB made substantial contributions to the conception and design of the study, helped to draft the manuscripts, reviewed and approved the submitted version. 


\section{Funding}

The NutBrain Study is supported by a grant of Ministero della Salute (Bando di Ricerca Finalizzata Giovani Ricercatori 2016, GR -2016-02361730). The study funders had no involvement in the design and conduct of the study, collection, management, analysis and interpretation of data; preparation, review, or approval of the manuscript; and the decision to submit the manuscript for publication.

\section{Availability of data and materials}

Data sharing is not applicable to this article as no datasets were generated or analysed during the current study.

\section{Ethics approval and consent to participate}

The study protocol and paperwork have been reviewed and approved by the Medical Ethical Committee of Pavia, Italy (Ref. number: 20180036036, 2018/04/20 and amendment Ref. Number: 20190045757, 2019/05/21). All participants provide a formal written informed consent in order to participate in the study according to the Declaration of Helsinki. In those individuals found to be without capacity to give full written informed consent, a caregiver or guardian is identified and their advice sought regarding participation.

\section{Consent for publication}

Not applicable.

\section{Competing interests}

The authors declare no competing interest.

\section{Author details}

${ }^{1}$ Institute of Biomedical Technologies-National Research Council, Via Fratelli Cervi, 9320090 Segrate, MI, Italy. ${ }^{2}$ IRCCS Mondino Foundation, Neuropsychology/Alzheimer's Disease Assessment Unit, Via Mondino 2, 27100 Pavia, Italy. ${ }^{3}$ Scuola Universitaria Superiore IUSS Pavia, Nets Center, Piazza della Vittoria, 15 -, 27100 Pavia, Italy.

Received: 16 December 2019 Accepted: 13 July 2020

Published online: 23 July 2020

\section{References}

1. Cevenini E, Bellavista E, Tieri P, Castellani G, Lescai F, Francesconi M, Mishto M, Santoro A, Valensin S, Salvioli S, et al. Systems biology and longevity: an emerging approach to identify innovative anti-aging targets and strategies. Curr Pharm Des. 2010;16(7):802-13.

2. http://www.un.org/en/development/desa/population/publications/pdf/ ageing/WPA2017_Report.pdf.

3. Brayne C, Miller B. Dementia and aging populations-a global priority for contextualized research and health policy. PLoS Med. 2017;14(3):e1002275.

4. Prince M, Ali GC, Guerchet M, Prina AM, Albanese E, Wu YT. Recent global trends in the prevalence and incidence of dementia, and survival with dementia. Alzheimers Res Ther. 2016;8(1):23.

5. Health WHODAphpGW, 2012. O.

6. Petersen RC, Caracciolo B, Brayne C, Gauthier S, Jelic V, Fratiglioni L. Mild cognitive impairment: a concept in evolution. J Intern Med. 2014;275(3): 214-28.

7. Ravaglia G, Forti P, Montesi F, Lucicesare A, Pisacane N, Rietti E, Dalmonte E, Bianchin M, Mecocci P. Mild cognitive impairment: epidemiology and dementia risk in an elderly Italian population. J Am Geriatr Soc. 2008:56(1):51-8.

8. Limongi F, Siviero P, Noale M, Gesmundo A, Crepaldi G, Maggi S. Dementia registry study G: prevalence and conversion to dementia of mild cognitive impairment in an elderly Italian population. Aging Clin Exp Res. 2017;29(3): 361-70.

9. Luck T, Luppa M, Briel S, Riedel-Heller SG. Incidence of mild cognitive impairment: a systematic review. Dement Geriatr Cogn Disord. 2010;29(2): $164-75$

10. Malek-Ahmadi M. Reversion from mild cognitive impairment to Normal cognition: a meta-analysis. Alzheimer Dis Assoc Disord. 2016;30(4):324-30.

11. Klimova B, Valis M, Kuca K. Cognitive decline in normal aging and its prevention: a review on non-pharmacological lifestyle strategies. Clin Interv Aging. 2017;12:903-10.
12. Morris MC. Nutrition and risk of dementia: overview and methodological issues. Ann N Y Acad Sci. 2016;1367(1):31-7.

13. Tucker KL. Nutrient intake, nutritional status, and cognitive function with aging. Ann N Y Acad Sci. 2016;1367(1):38-49.

14. Smith PJ, Blumenthal JA. Dietary factors and cognitive decline. J Prev Alzheimers Dis. 2016:3(1):53-64.

15. Tangney CC. DASH and Mediterranean-type dietary patterns to maintain cognitive health. Curr Nutr Rep. 2014;3(1):51-61.

16. Alles B, Samieri C, Feart C, Jutand MA, Laurin D, Barberger-Gateau P. Dietary patterns: a novel approach to examine the link between nutrition and cognitive function in older individuals. Nutr Res Rev. 2012;25(2):207-22.

17. van de Rest O, Berendsen AA, Haveman-Nies A, de Groot LC. Dietary patterns, cognitive decline, and dementia: a systematic review. Adv Nutr. 2015;6(2):154-68.

18. Shakersain B, Santoni G, Larsson SC, Faxen-Irving G, Fastbom J, Fratiglioni L, Xu W. Prudent diet may attenuate the adverse effects of Western diet on cognitive decline. Alzheimer's \& dementia : the journal of the Alzheimer's Association. 2016;12(2):100-9.

19. Ozawa M, Shipley M, Kivimaki M, Singh-Manoux A, Brunner EJ. Dietary pattern, inflammation and cognitive decline: the Whitehall II prospective cohort study. Clin Nutr. 2017;36(2):506-12.

20. Ozawa M, Ninomiya T, Ohara T, Doi Y, Uchida K, Shirota T, Yonemoto $K$, Kitazono T, Kiyohara Y. Dietary patterns and risk of dementia in an elderly Japanese population: the Hisayama study. Am J Clin Nutr. 2013; 97(5):1076-82.

21. Granic A, Davies K, Adamson A, Kirkwood T, Hill TR, Siervo M, Mathers JC, Jagger C. Dietary patterns high in red meat, potato, gravy, and butter are associated with poor cognitive functioning but not with rate of cognitive decline in very old adults. J Nutr. 2016;146(2):265-74.

22. Martinez-Lapiscina EH, Clavero P, Toledo E, Estruch R, Salas-Salvado J, San Julian B, Sanchez-Tainta A, Ros E, Valls-Pedret C, MartinezGonzalez MA. Mediterranean diet improves cognition: the PREDIMEDNAVARRA randomised trial. J Neurol Neurosurg Psychiatry. 2013;84(12) $1318-25$.

23. Valls-Pedret C, Sala-Vila A, Serra-Mir M, Corella D, de la Torre R, MartinezGonzalez MA, Martinez-Lapiscina EH, Fito M, Perez-Heras A, Salas-Salvado J, et al. Mediterranean diet and age-related cognitive decline: a randomized clinical trial. JAMA Intern Med. 2015:175(7):1094-103.

24. Moll van Charante EP, Richard E, Eurelings LS, van Dalen JW, Ligthart SA, van Bussel EF, Hoevenaar-Blom MP, Vermeulen M, van Gool WA: Effectiveness of a 6-year multidomain vascular care intervention to prevent dementia (preDIVA): a cluster-randomised controlled trial. Lancet 2016, 388(10046):797-805

25. Andrieu S, Guyonnet S, Coley N, Cantet C, Bonnefoy M, Bordes S, Bories L, Cufi MN, Dantoine T, Dartigues JF, et al. Effect of long-term omega 3 polyunsaturated fatty acid supplementation with or without multidomain intervention on cognitive function in elderly adults with memory complaints (MAPT): a randomised, placebo-controlled trial. Lancet Neurol. 2017;16(5):377-89.

26. Ngandu T, Lehtisalo J, Solomon A, Levalahti E, Ahtiluoto S, Antikainen R, Backman L, Hanninen T, Jula A, Laatikainen T, et al. A 2 year multidomain intervention of diet, exercise, cognitive training, and vascular risk monitoring versus control to prevent cognitive decline in at-risk elderly people (FINGER): a randomised controlled trial. Lancet. 2015;385(9984):2255-63.

27. Smith PJ, Blumenthal JA, Babyak MA, Craighead L, Welsh-Bohmer KA, Browndyke JN, Strauman TA, Sherwood A. Effects of the dietary approaches to stop hypertension diet, exercise, and caloric restriction on neurocognition in overweight adults with high blood pressure. Hypertension. 2010;55(6):1331-8.

28. Blumenthal JA, Smith PJ, Welsh-Bohmer K, Babyak MA, Browndyke J, Lin PH, Doraiswamy PM, Burke J, Kraus W, Hinderliter A, et al. Can lifestyle modification improve neurocognition? Rationale and design of the ENLIGH TEN clinical trial. Contemporary clinical trials. 2013;34(1):60-9.

29. Tussing-Humphreys L, Lamar M, Blumenthal JA, Babyak M, Fantuzzi G, Blumstein L, Schiffer L, Fitzgibbon ML. Building research in diet and cognition: the BRIDGE randomized controlled trial. Contemporary clinical trials. 2017:59:87-97.

30. 2013 WVEoDlotBiMCIM.

31. Horie N. Caloric Restriction in Obese Patients With Mild Cognitive Impairment: Effects on Adiposity $\mathrm{CaC}$. 
32. Lam V, Hackett M, Takechi R: Antioxidants and Dementia Risk: Consideration through a Cerebrovascular Perspective. Nutrients 2016, 8(12).

33. Morris MC. Symposium 1: vitamins and cognitive development and performance nutritional determinants of cognitive aging and dementia. Proc Nutr Soc. 2012;71:1-13.

34. Rogers GB, Keating DJ, Young RL, Wong ML, Licinio J, Wesselingh S. From gut dysbiosis to altered brain function and mental illness: mechanisms and pathways. Mol Psychiatry. 2016;21(6):738-48.

35. Scheperjans F, Aho V, Pereira PA, Koskinen K, Paulin L, Pekkonen E, Haapaniemi E, Kaakkola S, Eerola-Rautio J, Pohja M, et al. Gut microbiota are related to Parkinson's disease and clinical phenotype. Movement disorders : official journal of the Movement Disorder Society. 2015;30(3):350-8.

36. Manderino L, Carroll I, Azcarate-Peril MA, Rochette A, Heinberg L, Peat C, Steffen K, Mitchell J, Gunstad J. Preliminary evidence for an association between the composition of the gut microbiome and cognitive function in neurologically healthy older adults. Journal of the International Neuropsychological Society : JINS. 2017;23(8):700-5.

37. Bajaj JS, Ahluwalia V, Steinberg JL, Hobgood S, Boling PA, Godschalk M, Habib S, White MB, Fagan A, Gavis EA, et al. Elderly patients have an altered gut-brain axis regardless of the presence of cirrhosis. Sci Rep. 2016;6:38481.

38. Caracciolo B, Xu W, Collins S, Fratiglioni L. Cognitive decline, dietary factors and gut-brain interactions. Mech Ageing Dev. 2014;136-137:59-69.

39. Martin CR, Osadchiy V, Kalani A, Mayer EA. The brain-gut-microbiome Axis. Cell Mol Gastroenterol Hepatol. 2018;6(2):133-48.

40. Foster JA, Lyte M, Meyer E, Cryan JF: Gut Microbiota and Brain Function: An Evolving Field in Neuroscience. Int J Neuropsychopharmacol 2016, 19(5).

41. Tengeler AC, Kozicz T, Kiliaan AJ. Relationship between diet, the gut microbiota, and brain function. Nutr Rev. 2018;76(8):603-17.

42. Zamroziewicz MK, Barbey AK. Nutritional cognitive neuroscience: innovations for healthy brain aging. Front Neurosci. 2016;10:240.

43. Pistollato F, Cano SS, Elio I, Vergara MM, Giampieri F, Battino M. The use of neuroimaging to assess associations among diet, nutrients, metabolic syndrome, and Alzheimer's disease. Journal of Alzheimer's disease : JAD. 2015;48(2):303-18

44. Bonk J. A road map for the recruitment and retention of older adult participants for longitudinal studies. J Am Geriatr Soc. 2010;58(Suppl 2): S303-7

45. Craig $C L$, Marshall AL, Sjostrom M, Bauman AE, Booth ML, Ainsworth BE, Pratt M, Ekelund U, Yngve A, Sallis JF, et al. International physical activity questionnaire: 12-country reliability and validity. Med Sci Sports Exerc. 2003; 35(8):1381-95.

46. Nucci M, Mapelli D, Mondini S. Cognitive reserve index questionnaire (CRlq): a new instrument for measuring cognitive reserve. Aging Clin Exp Res. 2012; 24(3):218-26.

47. Lawton MP, Brody EM. Assessment of older people: self-maintaining and instrumental activities of daily living. The Gerontologist. 1969;9(3):179-86.

48. Katz S. Assessing self-maintenance: activities of daily living, mobility, and instrumental activities of daily living. J Am Geriatr Soc. 1983;31(12):721-7.

49. WC W: Nurses' Health Study Dietary questionnaire: appendix 5-1. In: In Nutritional Epidemiology, 2nd edition edn. Edited by Press. NYOU; 1998 pp. 95-97.

50. Albert MS, DeKosky ST, Dickson D, Dubois B, Feldman HH, Fox NC, Gamst A, Holtzman DM, Jagust WJ, Petersen RC, et al. The diagnosis of mild cognitive impairment due to Alzheimer's disease: recommendations from the National Institute on Aging-Alzheimer's Association workgroups on diagnostic guidelines for Alzheimer's disease. Alzheimer's \& dementia : the journal of the Alzheimer's Association. 2011;7(3):270-9.

51. Magni E, Binetti G, Bianchetti A, Rozzini R, Trabucchi M. Mini-mental state examination: a normative study in Italian elderly population. Eur J Neurol. 1996;3(3):198-202

52. Frasson P, Ghiretti R, Catricala E, Pomati S, Marcone A, Parisi L, Rossini PM, Cappa SF, Mariani C, Vanacore $N$, et al. Free and cued selective reminding test: an Italian normative study. Neurol Sci. 2011;32(6):1057-62.

53. Novelli G, Papagno, C., Capitani, E., and Laiacona, M. : Tre test clinici di ricerca e produzione lessicale. Taratura su sogetti normali. Archivio di psicologia, neurologia e psichiatria. In., edn.; 1986.

54. Caffarra P, Vezzadini G, Dieci F, Zonato F, Venneri A. Rey-Osterrieth complex figure: normative values in an Italian population sample. Neurol Sci. 2002; 22(6):443-7.

55. Appollonio I, Leone M, Isella V, Piamarta F, Consoli T, Villa ML, Forapani E, Russo A, Nichelli P. The frontal assessment battery (FAB): normative values in an Italian population sample. Neurol Sci. 2005;26(2):108-16.
56. Carlesimo GA, Caltagirone C, Gainotti G. The mental deterioration battery: normative data, diagnostic reliability and qualitative analyses of cognitive impairment. The Group for the Standardization of the mental deterioration battery. Eur Neurol. 1996;36(6):378-84.

57. Giovagnoli AR, Del Pesce M, Mascheroni S, Simoncelli M, Laiacona M, Capitani E. Trail making test: normative values from 287 normal adult controls. Ital J Neurol Sci. 1996;17(4):305-9.

58. Sartori G, and Job, R. : The oyster with four legs: A neuropsychological study on the interaction of visual and semantic information. Cognitive Neuropsychology. 1988 5((1):105-132.

59. LS. R: the CES-D scale: a self-report depression scale for research in the general population. . Appl Psychol Meas 1977, 1(3):385-401.

60. Guigoz Y, Vellas B. The mini nutritional assessment (MNA) for grading the nutritional state of elderly patients: presentation of the MNA, history and validation. Nestle Nutrition workshop series Clinical \& performance programme. 1999;1:3-11 discussion 11-12.

61. Petersen RC. Early diagnosis of Alzheimer's disease: is $\mathrm{MCl}$ too late? Curr Alzheimer Res. 2009;6(4):324-30.

62. Wenham PR, Price WH, Blandell G. Apolipoprotein E genotyping by onestage PCR. Lancet. 1991;337(8750):1158-9.

63. Consolandi C, Turroni S, Emmi G, Severgnini M, Fiori J, Peano C, Biagi E, Grassi A, Rampelli S, Silvestri E, et al. Behcet's syndrome patients exhibit specific microbiome signature. Autoimmun Rev. 2015;14(4):269-76.

64. Caporaso JG, Kuczynski J, Stombaugh J, Bittinger K, Bushman FD, Costello EK, Fierer N, Pena AG, Goodrich JK, Gordon Jl, et al. QIIME allows analysis of highthroughput community sequencing data. Nat Methods. 2010;7(5):335-6.

65. Wang Q, Garrity GM, Tiedje JM, Cole JR. Naive Bayesian classifier for rapid assignment of rRNA sequences into the new bacterial taxonomy. Appl Environ Microbiol. 2007;73(16):5261-7.

66. Lozupone C, Lladser ME, Knights D, Stombaugh J, Knight R. UniFrac: an effective distance metric for microbial community comparison. ISME J. 2011; 5(2):169-72.

67. Sachdev PS, Lipnicki DM, Kochan NA, Crawford JD, Thalamuthu A, Andrews G, Brayne C, Matthews FE, Stephan BC, Lipton RB, et al. The prevalence of mild cognitive impairment in diverse geographical and Ethnocultural regions: the COSMIC collaboration. PLoS One. 2015;10(11):e0142388.

68. Leite ML. Applying compositional data methodology to nutritional epidemiology. Stat Methods Med Res. 2016;25(6):3057-65.

69. Leite MLC, Prinelli F. A compositional data perspective on studying the associations between macronutrient balances and diseases. Eur J Clin Nutr. 2017.

70. Cornelis MC, Hu FB: Systems Epidemiology: A New Direction in Nutrition and Metabolic Disease Research. Curr Nutr Rep 2013, 2(4).

\section{Publisher's Note}

Springer Nature remains neutral with regard to jurisdictional claims in published maps and institutional affiliations.

\section{Ready to submit your research? Choose BMC and benefit from:}

- fast, convenient online submission

- thorough peer review by experienced researchers in your field

- rapid publication on acceptance

- support for research data, including large and complex data types

- gold Open Access which fosters wider collaboration and increased citations

- maximum visibility for your research: over $100 \mathrm{M}$ website views per year

At $\mathrm{BMC}$, research is always in progress.

Learn more biomedcentral.com/submissions 